\title{
Introduciendo el profesionalismo médico en etapas precoces del currículo de medicina: un curso para alumnos de primer año del Grado de Medicina
}

\author{
Jordi Palés-Argullós, Xavier Gasull-Casanova, David Soto, Núria Comes, Maria Nolla-Domenjó, Arcadi Gual
}

Resumen. La adquisición de valores y actitudes es un aspecto esencial de los resultados del aprendizaje de los estudiantes de medicina junto con los conocimientos y las habilidades prácticas y forma parte de lo que se conoce como profesionalismo médico. El proceso de aprendizaje de un médico implica no sólo el desarrollo de conocimientos y habilidades, sino también un proceso de transformación del alumno en el transcurso del cual aprende a ser diferente y a ser capaz de integrarse en una comunidad profesional. Por ello, debe considerarse seriamente la formación de nuestros estudiantes en este campo. Esta formación debe ser un proceso continuo y desde los primeros momentos del Grado de Medicina. En el contexto de la reforma de Bolonia, la Facultad de Medicina de la Universitat de Barcelona y otras facultades de medicina españolas han definido sus resultados de aprendizaje. Entre ellos, figura que los estudiantes deben ser capaces de aplicar los valores profesionales de excelencia como altruismo, compromiso, responsabilidad, integridad y honestidad en la práctica médica. Sin embargo, esta temática no está suficientemente representada en las actividades de aprendizaje del plan de estudios. Por ello, hace seis años, decidimos implementar un curso introductorio sobre profesionalismo médico dirigido a estudiantes de primer curso de medicina. En este trabajo describimos nuestra experiencia durante esos años. Los resultados de esta experiencia indican que la introducción del profesionalismo desde momentos iniciales del Grado de Medicina es factible y altamente apreciado por los estudiantes.

Palabras clave. Grado de Medicina. Profesionalismo.

Introducing medical professionalism in early moments of the medical curriculum: a course for first year's medical students

Summary. The acquisition of values and attitudes is an essential aspect of the learning outcomes of medical students together with knowledge and practical skills and they are part of what is known as medical professionalism. The learning process of a physician implies not only the development of knowledge and skills but also a transformational process of the learner, whereby he/she also learns to be different and to be able to join a professional community. Therefore we need to consider seriously the training of our students in this field. This training must be a continuous and on-going process throughout the entire educational continuum, beginning from early moments in medical school. In the context of the Bologna reform the Medical School of the University of Barcelona, and other Spanish medical schools, have defined their learning outcomes. Among them, the students must be able to apply the professional values of excellence, altruism, commitment, responsibility, integrity and honesty in medical practice, but this topic is insufficiently represented in the curriculum learning activities. Consequently six years ago, we decided to implement an introductory course on medical professionalism addressed to first year medical students. We describe our experience during the last six years of the implementation and development of this introductory course. Our results indicate that the introduction of professionalism from the earliest moments of the educative process in medical schools is feasible and is highly appreciated by our students.

Key words. Professionalism. Undergraduate medical curriculum.

\section{Introducción}

El profesionalismo se ha convertido en los últimos tiempos en un tema emergente en educación médica. Sin embargo no es un hecho tan nuevo. Si retrocedemos en el tiempo, el juramento hipocrático
(500 a.C.) y su versión moderna, la Declaración de Ginebra (1948) [1], ya hacen referencia a los atributos del profesionalismo y los valores que los médicos deberían tener. En su informe de 1910, Flexner [2] expresaba la necesidad de formar a los médicos en actitudes y valores. Un siglo más tarde, el nuevo
Departamento de Biomedicina; Facultad de Medicina y Ciencias de la Salud; Universitat de Barcelona (J. Palés-Argullós, X. Gasull-Casanova, D. Soto, N. Comes, A. Gual). Consell Català de Formació Continuada de les Professions Sanitàries (M. NollaDomenjó). Barcelona, España.

Correspondencia: Dr. Jordi Palés Argullós. Departamento de Biomedicina. Facultad de Medicina y Ciencias de la Salud. Universitat de Barcelona. Casanova, 143. E-08036 Barcelona.

E-mail:

jpales@ub.edu

Recibido:

14.02.17.

Aceptado:

15.02.17.

Conflicto de intereses: No declarado.

Competing interests: None declared.

(c) 2017 FEM 
informe Carnegie sobre educación médica [3] indicaba claramente que la adquisición de la identidad profesional y el desarrollo de valores profesionales deben ser la base de la educación médica, por lo que proponía reutilizar el concepto de profesionalismo como elemento clave de la educación de los médicos. La transformación personal que tiene lugar durante la formación de un médico requiere no sólo la adquisición de conocimientos formales y habilidades clínicas, sino también de valores.

Hoy día, los médicos se enfrentan a grandes cambios como las innovaciones tecnológicas, las diferentes formas de prestación de la atención sanitaria y las presiones económicas, entre otros. La educación médica debe prever que los estudiantes conozcan la existencia de dichos conflictos entre sus obligaciones tradicionales y los imperativos del mercado. En este contexto, los médicos que encarnen el ideario del profesionalismo estarán mejor preparados para comprender la dimensión social de su profesión [4].

En la actualidad, un nuevo concepto de profesionalismo médico está surgiendo no sólo porque haya cambiado en sí, sino también porque los cambios en el entorno plantean la urgente necesidad de repensar el compromiso social de los médicos. Ello se ha reflejado en los últimos años en diferentes documentos, especialmente el Physician Charter, propuesto por el American Board of Internal Medicine [5]. Por su parte, la formación en los valores de los profesionalismo debe ser continuado y constante a través del continuo educativo, empezando lo más precozmente posible y proveyendo actividades y oportunidades para el aprendizaje y la reflexión con el objetivo de adquirir estos valores [6].

En el contexto de la reforma propiciada por el proceso de Bolonia, la Facultad de Medicina de la Universitat de Barcelona implementó su nuevo plan de estudios en el año 2009, estableciendo los diferentes resultados de aprendizaje. Entre ellos, que los estudiantes deben conocer y aplicar los valores profesionales de excelencia, altruismo, responsabilidad, integridad y honestidad en su práctica médica reconociendo la necesidad de mantener la competencia profesional y respetar la autonomía de los pacientes, sus creencias y su cultura. En esta declaración formal, la facultad reconoce la importancia de los valores profesionales, pero la temática está insuficientemente representada en el plan de estudios. En consecuencia, decidimos implementar un curso de profesionalismo dirigido a alumnos de primer año del Grado de Medicina. En este artículo describimos esta experiencia y sus resultados.

\section{Diseño del curso e implementación}

El curso se dirige a alumnos de primer año del Grado de Medicina como una asignatura optativa. El número de alumnos se limita a un máximo de 20 . La asignatura tiene una duración de 75 horas y se desarrolla durante un semestre. Estas horas se distribuyen en actividades presenciales (40\%) y no presenciales basadas en el aprendizaje autónomo (60\%).

Los resultados del aprendizaje esperados al final de la asignatura son que el alumno conozca el concepto moderno del profesionalismo médico, que conozca y entienda los atributos del profesionalismo médico, que reconozca los valores profesionales implicados en diferentes situaciones reales y que reflexione sobre el papel del profesionalismo médico en la práctica médica diaria.

Las diferentes actividades de aprendizaje desarrolladas son:

- Lecciones teóricas (2-3 h), en las que se describe el concepto de profesionalismo y sus valores.

- Charlas de profesionales médicos invitados a los que se les pide que expliquen su visión del profesionalismo.

- Sesiones de trabajo en grupo en las que los estudiantes analizan y discuten diferentes documentos sobre la temática [6-8].

- Discusión en grupo entero de situaciones reales presentadas como viñetas y expuestas mediante role-playing por los propios estudiantes. En estas viñetas se presentan situaciones en las que están implicados diferentes atributos del profesionalismo. En la tabla I se exponen dos ejemplos de casos discutidos.

- Reflexión: debido a la importancia de la reflexión como instrumento para el aprendizaje del profesionalismo en diferentes sesiones, se estimula la reflexión sobre las conductas adecuadas en situaciones concretas.

\section{Evaluación del estudiante}

Los estudiantes son evaluados continuamente en base a su asistencia y su participación en las diferentes actividades y debates. Además, al final del curso, se proponen algunos casos prácticos para su análisis y reflexión. Finalmente, los estudiantes tienen que elaborar un portafolio personal con sus reflexiones sobre el curso y su experiencia de aprendizaje.

\section{Evaluación del curso}

Hemos evaluado la satisfacción del estudiante con el curso a través de una encuesta que utiliza una es- 
cala tipo Likert (0: totalmente en desacuerdo; 4: totalmente de acuerdo). La encuesta también contiene dos preguntas cerradas: ¿ha cambiado el curso su idea previa de lo que es un profesional médico?, ¿está dispuesto a realizar una nueva versión de la asignatura en años posteriores?

Además, existe un espacio para comentarios abiertos de los estudiantes.

\section{Resultados}

La encuesta se administra cada año a todos los estudiantes a final del curso. Los resultados se muestran en la tabla II. Los ítems más valorados en la encuesta fueron los relativos a la necesidad de abordar la temática del profesionalismo a lo largo del currículo médico en diferentes momentos (ítem 14: $3,9)$ y la utilidad de las presentaciones por diferentes profesionales (ítem 3: 3,8). El ítem con una valoración más baja fue el referente a la utilidad de las clases teóricas (ítem 2: 2,0).

Más de la mitad de los participantes (57\%) estuvieron de acuerdo o muy de acuerdo con que el curso había cambiado su idea de profesional médico. El 43\% indicó que su punto de vista no había cambiado o no lo sabía con seguridad. Casi todos los estudiantes (95\%) estaban dispuestos a realizar una nueva versión de la asignatura en años posteriores.

De los comentarios de los estudiantes se extraen los puntos fuertes y las acciones de mejora, que se indican en la tabla III.

\section{Discusión}

La principal ventaja de esta innovación ha sido la oportunidad de introducir el concepto de profesionalismo médico y sus valores a los jóvenes estudiantes y enfatizar la necesidad de cultivar esos valores a lo largo de su carrera y toda su vida profesional.

Los resultados de la encuesta indican que el curso sobre profesionalismo médico es valorado positivamente por los estudiantes, pero requiere algunos ajustes. Entre los diferentes ítems de la encuesta debemos subrayar los resultados obtenidos en la pregunta acerca de si las ideas previas sobre lo que es un profesional médico habían cambiado como consecuencia de haber cursado la asignatura. Los resultados podrían explicarse por el hecho de que muchos jóvenes estudiantes que entran en la facultad de medicina con 18 años llegan ya con una idea clara e idealista sobre los atributos deseables en los médicos y estas ideas no cambian significativamen-
Tabla I. Ejemplos de viñetas planteadas a los estudiantes para la discusión.

1. Un paciente habitual de tu consulta te solicita un justificante de una enfermedad inexistente para recuperar la penalización de una compañía aérea al no haber volado el día para el que adquirió los billetes

2. El representante de un laboratorio farmacéutico te visita en la consulta. Te propone asistir un fin de semana a una reunión en Londres con todos los gastos pagados, donde se presentará un nuevo fármaco

Tabla II. Encuesta de valoración de los estudiantes sobre diferentes aspectos del curso.

\begin{tabular}{|c|c|}
\hline & Media $\pm \mathrm{DE}$ \\
\hline He encontrado la asignatura muy interesante y útil para mi profesión & $3,7 \pm 0,30$ \\
\hline Las presentaciones teóricas han sido muy útiles & $2,0 \pm 0,89$ \\
\hline Las presentaciones de los diferentes profesionales han sido muy útiles & $3,8 \pm 0,43$ \\
\hline Las discusiones en clase han sido muy fructíferas & $3,4 \pm 0,64$ \\
\hline El trabajo en equipo ha sido muy útil & $3,4 \pm 0,71$ \\
\hline Los casos prácticos planteados han sido adecuados e interesantes & $3,4 \pm 0,60$ \\
\hline Las documentación facilitada ha sido interesante & $2,8 \pm 0,60$ \\
\hline Las sesiones de role-playing ha sido muy útiles & $3,6 \pm 0,80$ \\
\hline La atmósfera del curso ha sido muy buena & $3,6 \pm 0,60$ \\
\hline He disfrutado con la asignatura & $3,3 \pm 0,80$ \\
\hline Recomendaría a otros compañeros cursar la asignatura & $3,5 \pm 0,80$ \\
\hline La asignatura debería ser obligatoria para todos los estudiantes de la facultad & $2,9 \pm 1,20$ \\
\hline $\begin{array}{l}\text { El concepto de profesionalismo y sus valores deben ser } \\
\text { discutidos a lo largo de la carrera en varios momentos }\end{array}$ & $3,9 \pm 0,80$ \\
\hline He entendido el concepto de profesionalismo y sus valores & $3,8 \pm 1,00$ \\
\hline $\begin{array}{l}\text { En caso de que mi idea de profesionalismo fuera diferente, } \\
\text { la asignatura me ha cambiado la idea de lo que es un profesional médico }\end{array}$ & $2,7 \pm 1,40$ \\
\hline Valoración global & $49 / 60(81 \%)$ \\
\hline
\end{tabular}

Puntuación de 1 a 4 (1: totalmente en desacuerdo; 4: totalmente de acuerdo). DE: desviación estándar.

te después de cursar la asignatura, al menos en la mitad de los encuestados.

Por otra parte, puede sorprender la baja puntuación referente a las clases teóricas, sobre todo si tenemos en cuenta que ocupan una parte mínima del tiempo. Sin embargo, consideramos que dado que se trata de estudiantes de primer curso, acostum- 
Tabla III. Puntos fuertes y aspectos de mejora aportados por los estudiantes.

\begin{tabular}{ll}
\hline La asignatura me ha ayudado a reflexionar sobre el concepto de profesionalismo \\
Puntos fuertes & $\begin{array}{l}\text { El dinamismo de la asignatura } \\
\text { La importancia dada al compromiso ético y moral del médico }\end{array}$ \\
\hline Aspectos de mejora & $\begin{array}{l}\text { Incrementar el número de situaciones prácticas para discutir } \\
\text { Incrementar los ejercicios de role-playing }\end{array}$ \\
\hline
\end{tabular}

brados hasta el momento a cursar casi exclusivamente temáticas del ámbito biomédico y que no han entrado en contacto con anterioridad con el tema del profesionalismo, se hace necesario invertir un tiempo mínimo en explicar el concepto de profesionalismo y describir claramente los objetivos del curso.

Una dificultad importante que hemos apreciado en la implementación del curso ha sido el carácter innovador de la propuesta. Aunque el concepto de profesionalismo es bien conocido por los profesores, especialmente si son médicos, éstos no tienen experiencia en la enseñanza de tales aspectos y en ocasiones se sienten desorientados de cómo enfocar su docencia. Por ello se hace necesario adquirir alguna experiencia en la enseñanza y el aprendizaje de profesionalismo por parte del profesorado.

Consideramos como dato muy positivo que el 95\% de los estudiantes estaría dispuesto a realizar una nueva versión del curso en años posteriores, adaptada a las necesidades específicas de estudiantes 'sénior'. Por ello pensamos que seria necesario extender este curso a todos los estudiantes y revisar el tema para los estudiantes a lo largo de todo el grado.

En conclusión, podemos afirmar que la introducción del profesionalismo, desde las primeras etapas de la educación médica es factible y altamente recomendable. También es necesario revisar periódicamente este tema a lo largo del currículo. Nuestra experiencia, la primera de una facultad de medicina española, podría servir de ejemplo a otras facultades de medicina y actuar de catalizador para la introducción de cursos similares.

\section{Bibliografía}

1. World Medical Organization. Declaration of Geneva. Adopted by the General Assembly of World Medical Association at Geneva Switzerland, September 1948. URL: http://www.cirp. org/library/ethics/geneva/.

2. Flexner A. Medical education in the United States and Canada: a report to the Carnegie Foundation for the Advancement of Teaching. Bulletin no. 4. New York: Carnegie Foundation for the Advancement of Teaching; 1910.

3. Cooke M, Irby DM, O’Brien BC. Educating physicians: a call for reform of medical school and residency. San Francisco, CA: Jossey-Bass; 2010.

4. Pardell H, ed. El médico del futuro. Barcelona: Fundación Educación Médica; 2009.

5. ACP-ASIM, ABIM Foundation, EFMI. Medical professionalism in the new millennium: a physician charter. Lancet 2002; 359: 520-2.

6. Goldie J, Dowie A, Cotton P, Morrison J. Teaching professionalism in the early years of a medical curriculum: a qualitative study. Med Educ 2007; 41: 610-7.

7. Royal College of Physicians. Doctor in society. Medical professionalism in a changing world. Clin Med 2005; 5 (Suppl 1): S5-40.

8. General Medical Council. Medical students professional values and fitness to practice. Manchester, UK: General Medical Council; 2009. URL: http://www.gmc-uk.org/Medical_students professional_values_and_fitness_to_practise_1114.pdf_ 48905163.pdf.

9. Cruess RL, Cruess SR, Steinert Y, eds. Teaching medical professionalism. Cambridge: Cambridge University Press; 2009. 